\title{
Impaired Sleep-Related Learning in Children with Williams Syndrome
}

\author{
Dagmara Dimitriou ${ }^{1}$, Annette Karmiloff-Smith ${ }^{2}$, Anna Ashworth ${ }^{1}$ \\ and Catherine M. Hill \\ ${ }^{1}$ Department of Psychology \& Human Development, Institute of Education, London, UK \\ ${ }^{2}$ Birkbeck Centre for Brain and Cognitive Development, University of London, UK \\ ${ }^{3}$ Division of Clinical Experimental Sciences, Faculty of Medicine, University of Southampton, UK \\ Correspondence should be addressed to: Dagmara Dimitriou; d.dimitriou@ioe.ac.uk
}

Received 4 February 2013; Accepted 11 March 2013; Published 24 April 2013

Academic Editor: Miho Nakamura

Copyright (C) 2013 Dagmara Dimitriou, Annette Karmiloff-Smith, Anna Ashworth and Catherine M. Hill. Distributed under Creative Commons CC-BY 3.0

\begin{abstract}
Recent research has established that sleep is essential for memory consolidation in learning and academic performance of children and adults. Similar evidence in childhood is emerging. Conversely, sleep deprivation and/or sleep problems usually weaken these functions. The present study investigates the association between sleep related learning in school-aged children with Williams syndrome $(\mathrm{N}=14)$ compared to the typically developing children $(\mathrm{N}=14)$. Sleep was measured using actigraphy and parents completed the Child Sleep Health Questionnaire. Accuracy performance on a well-characterised procedural learning task- the finger tapping motor sequence task (FTT) was assessed on three sessions. Children in the typically developing group showed increased accuracy scores following a period of sleep (14\% improvement) compared with wake (drop of $2 \%$ ). In contrast children with Williams syndrome showed an initial improvement in accuracy on the training session, albeit slow, but their accuracy deteriorated following a period of sleep. The sleep-related decrement in performance on the procedural task may reflect sleep problems that are now well characterised in the WS group. This study demonstrated the contribution of sleep to procedural learning in typically developing children. Further studies may elucidate the reasons why similar sleep related benefits are not seen in children with Williams syndrome. Meanwhile practitioners and families should ensure that children obtain adequate sleep in order to maximise their attention readiness to learn and achieve optimum cognitive performance.
\end{abstract}

Keywords: Sleep, Williams syndrome, finger Tapping, procedural learning, actigraphy.

Cite this Article as: Dagmara Dimitriou, Annette Karmiloff-Smith, Anna Ashworth and Catherine M. Hill (2013), "Impaired Sleep-Related Learning in Children with Williams Syndrome," Short Title: "Sleep and Learning in Williams Syndrome," Pediatrics Research International Journal, Vol. 2013 (2013), Article ID 662275, DOI: $10.5171 / 2013.662275$ 


\section{Introduction}

Sleep is essential for healthy brain development. It represents the major state of the brain in early childhood, suggesting a functional role in neurodevelopment. Neurocognitive research in sleep-deprived adults indicates a negative impact on mood, cognitive performance, and motor function. Cognitive domains such as executive attention and working memory are particularly susceptible (Ashworth, Hill, Karmiloff-Smith and Dimitriou, 2013; Durmer and Dinges, 2005). Sleep loss may be socially imposed or may result from underlying sleep disorders such as periodic limb movement disorder of sleep apnoea that cause 'sleep fragmentation' as the sleep is punctuated with numerous arousals.

Similar findings have been obtained in the few experimental studies carried out on children, when exposed to very marginal sleep restriction. For instance, Sadeh, Gruber and Raviv (2003) investigated the neurobehavioural effects of extending or restricting 9 and 11 year old children's usual sleep patterns by one hour per night for three consecutive nights. The authors examined performance profiles using tasks such as working memory, motor speed and reaction time, vigilance, visual memory, and attention. Children adjusted their sleep by an average of 35 minutes in the sleep extension group and by 41 minutes in the sleep restriction group. Surprisingly, sleep quality improved in the sleep-restricted group, whereas the opposite occurred in the extension group. Children in the sleep extended-sleep group improved their performance on continuous performance reaction time and digit span forward, whilst the sleep restriction group showed no change on these tasks. The neurocognitive improvements seen after a 35 minutes extension in sleep are comparable to those gained by two years of development, showing how sensitive children are to modest alterations of their natural sleep patterns.
Importantly, these laboratory findings translate into real world settings. Metaanalysis of the effects of sleep quality, sleep duration and daytime sleepiness on school performance in healthy TD children and adolescents (Dewald, Meijer, Oort, Kerkhof and Bögels, 2010) showed that all sleep dimensions were significantly associated with school performance. Similarly, Ashworth and colleagues (2013) reported that after sleep period, TD children showed significant improvements in performance of $14 \%$ on a declarative learning task and $25 \%$ on a procedural learning task, but no significant change in score following a wake retention interval.

In sum, recent data have revealed that sleep not only enhances daytime cognition and learning but, importantly, it also plays an active role in learning. Indeed, a large number of sleep related studies have shown to have beneficial effects on both declarative (Gais et al., 2009) and procedural learning tasks in adults (Brawn, et al., 2010). Most robust and reliable sleep-dependent gains in performance have been revealed on a simple, well-characterised test of motor skill learning, the Finger Tapping Motor Sequence Test (e.g., Karni et al., 1995; Walker, Brakefield, Morgan, Hobson, and Stickgold 2002). Speed and accuracy have been found to improve over time, especially when retesting occurred following a period of sleep (18.9\% improvement) compared with wake (3.9\% improvement). Gains in accuracy occurred regardless of whether initial training took place in the morning or in the evening. Additional nights of sleep led to further improvement, in the absence of further exposure to the task (Walker et al 2003), whereas sleep deprivation the first night after training blocked subsequent practice-independent improvement (Fischer et al 2002; Walker et al 2002). These findings demonstrate that overnight improvement on this task depends on sleep rather than the mere passage of time. 
Very few studies into sleep-dependent procedural memory consolidation have been carried out in children. The few that have contrast with the adult findings in that sleep learning outcomes are mixed, with some studies indicating that procedural memories are not consolidated during sleep in children (e.g., Prehn-Kristensen et al., 2009; Fischer, Wilhelm, and Born (2007); Wilhelm et al. (2008). For instance, using a mirror-tracing task in children aged 10 to 13 years, PrehnKristensen and others reported that performance improved due simply to practice effects but that it was not sleep dependent. The authors proposed that during childhood, procedural learning tasks have to compete with overall learning for off-line consolidation and so do not improve following sleep. In contrast, Dorfberger, AdiJapha and Karni (2007) tested 9, 12 and 17 year olds on the FTT (experiment 1) and reported performance gains after sleep period comparable to those reported for adults. These inconsistent findings are probably due to methodological differences as well as to failure to take into account developmental changes in sleep architecture and learning profiles (Annaz, Karmiloff-Smith and Thomas, 2008).

It is noteworthy that sleep disturbances are common in children with neurodevelopmental disorders such as Autism, ADHD, Down syndrome and Williams syndrome. It is therefore possible that sleep disturbances contribute to some of the cognitive difficulties/impairments observed in these children, which could be improved if sleep problems were treated. We thus examined sleep-dependent learning in one of these disorders, Williams syndrome (WS). WS is a rare genetic disorder caused by a hemizygous microdeletion of some 28 genes on chromosome 7q11.23 (Pober, 2010) with an incidence of approximately 1 in 20,000 live births (Morris, Demsey, Leonard, Dilts and Blackburn, 1988). The main cognitive characteristics of WS include full IQ levels ranging from 40 to 90 , with the majority scoring between 55 and 69 (Mervis et al., 2000; Searcy et al., 2004), relatively proficient face recognition and language skills, and poor visuo-spatial and numerical skills (Donnai and Karmiloff-Smith, 2000; Mervis and Bertrand, 1997).

Only a handful of studies have examined sleep in WS but all reported sleep disturbances (e.g., Annaz, 2011; Arens et al., 1998; Goldman, Malow, Newman, Roof and Dykens, 2009). More recently, a polysomnographic (PSG) study by Mason and colleagues showed that individuals with WS (age range: 2- 18 years, $\mathrm{N}=35$ ) spent a greater percentage of the sleep period in a wake state and lower sleep efficiency. In other words, the percentage of time from sleep onset to morning waking that is actually spent asleep in WS is significantly reduced compared to typically developing (TD) children (Mason et al., 2011). This is in line with previous smaller PSG studies (Bódizs, Gombos and Kovács, 2009, Arens et al., 1998). The authors of these two earlier studies also observed increased uni- or bilateral leg movements, largely during NonREM sleep, and concluded that frequent periodic leg movements lead to sleep disruption in this group. Studies using parental reports and actigraphy give further support to the PSG findings, indicating that toddlers with WS had a shorter night's sleep, more night wakings and wakefulness (Axelsson et al., submitted), with long sleep latencies (defined as time to fall asleep from 'lights out'), sleep anxiety, bedtime resistance and night wakings throughout childhood (Annaz et al., 2011; Ashworth et al., 2013). To our knowledge, there have hitherto been no studies investigating the impact of these sleep disturbances on sleep-dependent consolidation of learning in WS.

In the current study, we examined sleeprelated learning with the Finger Tapping Task. Sleep quality and duration was measured using actigraphy, as well as a sleep diary and the parent completed Child Sleep Habits Questionnaire (CSHQ) (Owens, Spirito and McGuinn, 2000) was used to screen for common sleep disorders. Since the FTT is easy to carry out and requires very little 
instruction, it was deemed appropriate to use with children with a neurodevelopmental disorder. The present study aims to compare sleep-related performance improvements in children with WS to their comparison TD children.

\section{Methods}

\section{Participants}

Fourteen children with WS (5 male, 9 female; mean age $=8.9$ years) and 14 TD comparison group children, matched for chronological age and gender, participated in the study. Children with WS had been diagnosed clinically, as well as by means of the fluorescence in situ hybridisation test for the deletion of one copy of the Elastin gene. All individuals had normal or corrected-tonormal vision and were right handed. Children with WS were identified from the WS Foundation UK database, with TD children recruited from the North London schools. Children were not included in the study if they had co-morbid medical or psychiatric disorders and conditions that could affect sleep such as epilepsy, problems with tonsils/adenoids, frequent sinus infection and poorly controlled asthma or eczema; if they were taking any hypnotic medication.

Environmental factors such as whether children were watching TV 30 min before sleep, shared bedroom and if the child's bedroom on a main road where traffic can be heard at night were measured. No statistically significant difference was observed between groups in any of these parameters.

All participants were tested for nonverbal Mental Age using Raven's Progressive Matrices (Raven et al., 1990), and verbal Mental Age using the British Picture Vocabulary Scale II (Dunn et al., 1997). Participant characteristics are provided in Table 1. The experimental protocol was approved by the Institute of Education University of London Ethics Committee and the WS Foundation UK.
There was no difference between the groups in chronological age $(\mathrm{t}(28)=2.61, p=.32$ ). Next, in order to assess demographic differences between groups, a priori Chisquare analyses were conducted. The two groups did not differ on gender, $\chi 2(1)=1.70$, $p=.424$; ethnicity, $\chi 2(3)=4.11, p=.381$, parent occupation (professional, managerial and other occupation) $\chi 2(3)=4.46, p=.487$ and maternal education, $\chi^{2}(3)=4.59, p=.15$.

\section{Procedure}

\section{Finger Tapping Task}

All children completed a computerized version of the Finger Tapping Task, a motor memory task, in Microsoft Excel. A fivefigure target sequence (e.g., 4-1-3-2-4) was displayed at the top of the screen throughout. Children placed the four fingers of their left hand over the numbers one to four on the computer keyboard and were asked to repeatedly tap the target sequence, pressing the 'Enter' key with their right hand after each sequence.

The typed sequence was not displayed in the cells. Children tapped the target sequence as many times as possible during 30-second runs, with each run followed by a 30 -second rest period. The training phase consisted of 12 runs and took place in the evening within the two hours preceding the child's bedtime. The retest with 6 runs under the same conditions was performed the following morning before engaging in any other cognitive tasks, and a further retest of six trials was performed that same afternoon. All children were tested at home during the weekdays. No feedback was provided but children were offered a reward for their participation.

\section{Sleep Measures}

Actigraphy data was collected from each child for seven consecutive days using an Actiwatch Mini (Cambridge Neurotechnology, Cambridge, UK), a small activity-monitoring device worn on the non-dominant wrist. Sleep scoring covered 1-minute epochs, and 
raw data values were scored using Sleep Analysis 7 programme (CamNTech, Cambridge, UK). Data for each child were averaged over the seven nights, as recommended (Acebo et al. 1999). Variables of interest included: (1) sleep onset time, (2) assumed sleep time, (3) sleep percent (4) actual sleep time-sleep time excluding all periods of wakefulness, (5) sleep latency, (6) number of night-wakings, (7) moving minutes and (8) fragmentation (an indication of the degree of fragmentation of the sleep period, used as an indication of sleep quality). In addition, each parent was asked to complete a sleep diary to document bedtime and wake up time for the purposes of actigraphy analysis.

The Child Sleep Habits Questionnaire: Parents completed the Child Sleep Habits Questionnaire (CSHQ), a screening instrument for school-aged children based on common clinical symptom presentations of prevalent sleep disorders. The CSHQ consists of 33 items rated on a 3-point Likert scale (never/rarely, sometimes, and usually). It yields scores on 8 sub-scales: Sleep Anxiety (e.g., "afraid of sleeping in the dark"), Sleep Duration, Bedtime Resistance, Sleep Onset Delay, Night Waking, Parasomnias, Sleep Disordered Breathing and Daytime Sleepiness. It provides a total sleep disturbance score, as well as a problemspecific breakdown. Psychometric properties indicate that the CSHQ can identify sleep problems in a clinical sample and can distinguish between clinical and community samples (Marcus et al., 2000; Waumans, Terwee, Van den Berg, Knol, Van Litsenburg, Gemke, 2010).

\section{Results}

Data were analysed in SPSS v.18. ANOVA and multivariate ANCOVA comparisons were used. Data were checked using Levene's homogeneity of variance test (Levene's Test) for different groups, and for the covariance structure (Box's M test). In addition, the absence of outliers was assured before analyses were performed (see, Thomas, Annaz, Ansari, Scerif, Jarrold, and KarmiloffSmith, 2009, for details on analysis).

\section{Accuracy on Finger Tapping Task}

In line with previous studies, children in the TD group showed a significant improvement in task accuracy following sleep $(F(1,13)$ $=42.58, \mathrm{p}=.001)$. There was no significant further improvement following a further period of wake (p>.05) (see Figure 1). In contrast, children in the WS group did not show improvement following sleep $(F(1,13)$ $=2.38, \mathrm{p}=.15$ ) nor on the subsequent afternoon session $(\mathrm{F}(1,13)=4.38, \mathrm{p}=.35)$. Comparison of the performance of the control TD group with the WS group showed a significant difference on the task before and after sleep sessions $(F(1,26)=32.24, p=$ .001) whereby children in the WS group did not improve on the task following sleep. Error analyses of the TD group revealed a significant reduction in error rate following sleep $(\mathrm{F}(1,13)=38.58, \mathrm{p}=.001)$. In contrast, children in the WS group showed no difference in error rate across all the sessions and remained less accurate than the TD group (all $\mathrm{p}>0.5$ ). 


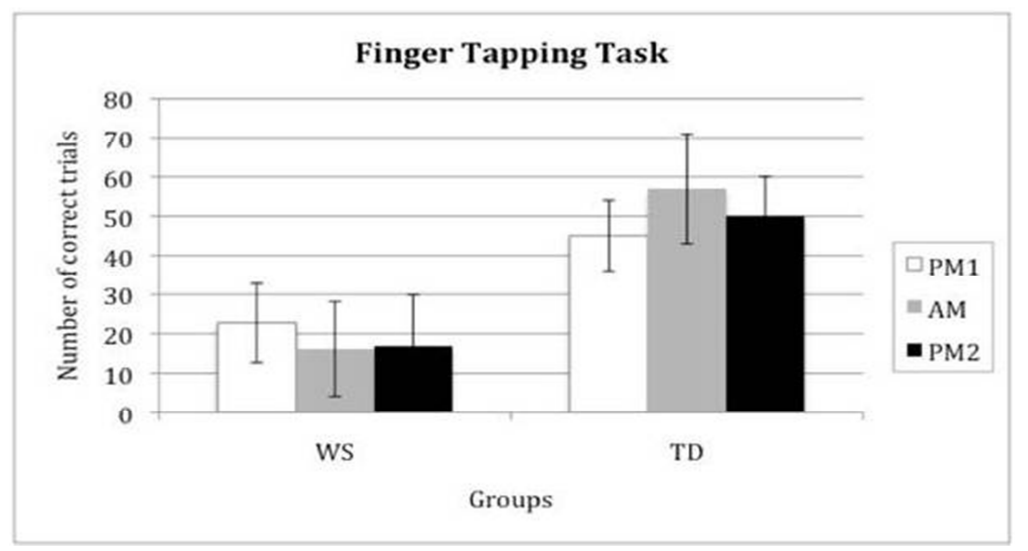

Figure 1: Accuracy Performance of the WS and TD Groups on the FFT Task

Since children in the WS group did not show any accuracy gains on the task, we examined each child's trajectories on every session. The results revealed that children in the WS group showed a very slow improvement on later trials, albeit not significant, but their morning performance reverted to the performance seen in the first trials of the evening session (all $\mathrm{p}>0.5$ ).

Table 1. Test Results per Group

\begin{tabular}{|c|c|c|c|c|}
\hline $\begin{array}{c}\text { Group } \\
\text { (sample size) }\end{array}$ & Statistic & CA (months) & Non-verbal MA & Verbal MA \\
\hline TD & Mean & 106 & 114 & 115 \\
\hline (n=14) & Std & 12 & 21 & 31 \\
\hline & Min & 73 & 102 & 108 \\
\hline & Max & 131 & 138 & 154 \\
\hline WS & Mean & 106 & 38 & 99 \\
\hline (n=14) & Std & 14 & 12 & 23 \\
\hline & Min & 74 & 36 & 86 \\
\hline & Max & 135 & 48 & 124 \\
\hline
\end{tabular}

$\mathrm{TD}=$ typically developing, WS = Williams syndrome. $\mathrm{CA}=$ chronological age, Non-verbal MA derived from the Raven's Coloured Progressive Matrices (Raven et al., 1990), Verbal MA derived from BPVS = British Picture Vocabulary Scale (Dunn et al., 1996).

\section{Comparison of WS and TD on Sleep Measures}

ANOVA analyses of group comparisons were performed to investigate if there were any differences between the WS and TD control group in the objective (actigraphy) and subjective (CSHQ) sleep measures. Actigraphy data revealed that children in the WS group had more problems than controls with sleep onset delay, moving minutes and fragmentation, indicating poor continuity of sleep. Based on the CSHQ scores, children with WS had greater difficulties with sleep anxiety, sleep onset delay, frequent night waking compared to the TD group. Notably, despite consistency across the measures of greater problem with sleep onset delay, the children with WS did not have greater bedtime resistance, suggesting this was unlikely to be a behavioural difficulty. 
Table 2. WS and TD Group Comparisons on Sleep Measures Using ANOVA Test

\begin{tabular}{|l|l|l|l|l|}
\hline $\begin{array}{l}\text { Actigraphic sleep measures } \\
\text { (weekdays data) }\end{array}$ & TD & WS & $F$ & $p$ \\
\hline Bed time (hh: mm) & $21: 14(0: 28)$ & $20: 43(0: 39)$ & 22.07 & .001 \\
\hline Assumed sleep time (hh: mm) & $9: 28(0: 39)$ & $9: 23(1: 04)$ & 3.98 & .09 \\
\hline Sleep efficiency (\%) & $88.93(3.16)$ & $88.59(3.19)$ & 3.67 & .07 \\
\hline Actual sleep time (hh: mm) & $08: 27(00: 22)$ & $08: 25(00: 43)$ & 1.19 & .09 \\
\hline Sleep latency (hh: mm) & $0: 27(0: 17)$ & $\mathbf{0 : 4 9 ( 0 : 3 9 )}$ & 18.51 & $\mathbf{. 0 0 1}$ \\
\hline Night wakings (\%) & $30.23(7.38)$ & $28.79(6.14)$ & 11.04 & .09 \\
\hline Moving minutes (hh: mm) & $72.31(23.27)$ & $\mathbf{8 4 . 0 0 ( 2 2 . 0 3 )}$ & 22.05 & $\mathbf{. 0 0 1}$ \\
\hline Fragmentation (\%) & $27.12(7.73)$ & $\mathbf{3 1 . 2 7}(\mathbf{6 . 6 5 )}$ & 23.09 & $\mathbf{. 0 0 1}$ \\
\hline CSHQ (possible score) & & & & \\
\hline Total Score (33-99) & $39.0(5.7)$ & $\mathbf{4 4 . 2 ( 5 . 7 )}$ & 27.12 & $\mathbf{. 0 0 1}$ \\
\hline Bedtime Resistance (6-18) & $6.7(1.7)$ & $7.0(1.5)$ & 4.34 & $>.5$ \\
\hline Sleep Onset Delay (1-3) & $1.3(0.5)$ & $1.8(0.8)$ & 27.18 & .001 \\
\hline Sleep Duration (3-9) & $3.1(0.5)$ & $3.8(1.1)$ & 3.11 & $>.5$ \\
\hline Sleep Anxiety (4-12) & $4.5(1.1)$ & $5.3(1.7)$ & 3.12 & $>.5$ \\
\hline Night Waking (3-9) & $3.5(0.8)$ & $\mathbf{4 . 5 ( 1 . 4 )}$ & 33.25 & $\mathbf{. 0 0 1}$ \\
\hline Parasomnias (7-21) & $8.1(1.0)$ & $\mathbf{9 . 0 ( 1 . 3 )}$ & 26.13 & $\mathbf{. 0 0 1}$ \\
\hline SDB (3-9) & $3.0(0.2)$ & $\mathbf{3 . 6 ( 0 . 9 )}$ & 35.34 & $\mathbf{. 0 0 1}$ \\
\hline Daytime Sleepiness (8-24) & $11.5(2.7)$ & $11.5(2.6)$ & 3.05 & $>.5$ \\
\hline
\end{tabular}

Note. Values are group weekly means (averaged across weekdays) and standard deviation.

\section{Finger Tapping Task and Sleep Measures}

For the WS group, ANCOVA analysis revealed that only some CSHQ subscales predicted performance scores on the FTT on the first afternoon session. These were: bedtime resistance $(F(1,13)=12.93, p=.006)$ and sleep disordered breathing $(\mathrm{F}=(1,13) 5.15, \mathrm{p}$ $=.049)$, as well as borderline significance with the CSHQ total scores $(\mathrm{F}(1,13)=4.78, \mathrm{p}$ $=.057)$. Only 'moving minutes' measured by actigraphy showed a significant link with the morning performance $(F(1,13)=6.41, p=$ .038). Task performance was not related to CSHQ scores nor actigraphic variables in the TD group.

\section{Discussion}

In line with previous studies (e.g., Karni et al., 1994, Walker et al., 2002), we found a significant overnight improvement in a procedural learning task in TD children following a period of sleep. Although our study demonstrated this effect only at the behavioural level, sleep-specific reorganization of skill representations in the brain has been demonstrated in studies using functional magnetic brain imaging (Maquet et al., 2003; Plihal and Born, 1997; Walker et al., 2005), so it is highly likely that the sleepdependent improvements resulted in neural changes too. Indeed, in these neural studies which also used a FFT task, at a post-sleep retesting participants showed less involvement of prefrontal, premotor, and primary motor cortical areas, alongside a stronger involvement of left parietal cortical regions and the basal ganglia, suggesting increased automatisation of performance after sleep. In contrast, such sleep-associated gains in performance were not seen in our WS group.

Similarly to previous reports, children with WS had increased levels of sleep disturbance, 
which may have contributed to the lack of overnight enhancement of task performance. One potential explanation for the failure of WS children to demonstrate sleep-related task improvement may lie in the nature of the fine motor skills required to perform the finger-tapping task in the first place. Also, the extent these improvements reflect actual performance 'gains' induced by sleep remains controversial, because the measured gains depend on the pre-sleep performance used as a reference, which itself can be subject to a number of factors. Poorer performance of the WS group might also be associated with increased levels of evening cortisol and reduced levels of melatonin which may result in delayed sleep onset in WS (Dimitriou, Sniecinska and Iles, 2013; Sniecinska et al., accepted). Sniecinska and colleagues tested 27 school-aged children with WS and matched TD children for levels of hormonal sleep markers such as melatonin and cortisol. Interestingly, they found abnormally higher levels of cortisol before bedtime and lower melatonin levels compared to the TD controls.

\section{Limitations}

Although we controlled for time of day effects on learning, it is possible that children with WS had undetected differences in their circadian physiology which impaired their learning capacity before bedtime relative to their TD peers. One way of addressing this in future studies would be to introduce a balanced design where half the group learned the task in the morning and were re-tested before and after sleep whilst the other half learned in the late afternoon before sleep and were tested at 2 intervals separated by a period of wake. Lastly, children with developmental delay such as WS may also consolidate memories differently to TD children, and a lack of sleep-related learning gains could contribute to learning difficulties (see Gervan, Gombos and Kovacs, 2012). Further research with larger groups and different tasks is necessary to elucidate whether offline learning occurs in these groups as it does in TD children.
In summary, the current study supports the notion that sleep is necessary for enhanced memory consolidation in TD children. This has far-reaching implications for child development hence sleep management ought to be targeted very early on as might result in improved learning (Jan et al., 2008; Richdale and Wiggs, 2005). Critically parents, teachers and clinicians need to recognise the importance ofsleep for cognitive health and development.

\section{Acknowledgements}

We would like to thank the Williams Syndrome Foundation, UK for putting us in touch with families. We are grateful to all children and teachers from the London schools for their continuing collaboration. This research was supported by the British Academy grant.

\section{References}

Acebo, C., Sadeh, A., Seifer, R., Tzischinsky, O., Wolfson, A. R., Hafer, A. \& Carskadon, M. A. (1999). "Estimating Sleep Patterns with Activity Monitoring in Children and Adolescents: How Many Nights are Necessary for Reliable Measures?," Sleep, 22(1) 95-103.

Annaz, D., Hill, C. M., Ashworth, A., Holley, S. \& Karmiloff-Smith, A. (2011). "Characterisation of Sleep Problems in School-Aged Children with Williams Syndrome," Research in Developmental Disabilities, 32(1) 164-169.

Annaz, D., Karmiloff-Smith, A. \& Thomas, M. C. S. (2008). The Importance of Tracing Developmental Trajectories for Clinical Child Neuropsychology, In Reed, J., and Warner Rogers, J. (Eds.), Child Neuropsychology: Concepts, Theory and Practice. Singapore: Wiley-Blackwell.

Arens, R., Wright, B., Elliott, J., Zhao, H., Wang, P. P., Brown, L. W., Namey, T. \& Kaplan, P. (1998). "Periodic Limb Movement in Sleep in Children with Williams Syndrome," The Journal of Pediatrics, 133(5) 670-674. 
Ashworth, A., Hill, C. M., Karmiloff-Smith, A. \& Dimitriou, D. (2013). "Cross Syndrome Comparison of Sleep Problems in Children with Down Syndrome and Williams Syndrome," Research in Developmental Disabilities, 34, 1572-1580.

Ashworth, A., Hill, C. M., Karmiloff-Smith, A. \& Dimitriou, D. (In Press) 'Sleep Enhances Learning in Children,' Journal of Child Experimental Psychology.

Axlesson, E., Sadeh, A., Hill. C. M. \& Dimitriou, D. (Under Review) 'Sleep and Related Factors in Toddlers with Williams Syndrome,'

Brawn, T. P., Nusbaum, H. C. \& Margoliash, D. (2010). "Sleep-Dependent Consolidation of Auditory Discrimination Learning in Adult Starlings," Journal of Neuroscience, 30(2) 609-613.

Bódizs, R., Gombos, F. \& Kovács, I. (2009). 'Sleep in Williams Syndrome: From Periodic Leg Movements to Architectural, EEG Spectral and Spindling Peculiarities' Abstracts of 3rd International Congress of the Association of Sleep Medicine (WASM), 10(2) 48-49.

Dewald, J. F., Meijer, A. M., Oort, F. J., Kerkhof, G. A. \& Bögels, S. M. (2010). "The Influence of Sleep Quality, Sleep Duration and Sleepiness on School Performance in Children and Adolescents: A Meta-Analytic Review," Sleep Medicine Reviews, 14(3), 179-189.

Dimitriou, D., Sniecinska, A. \& Iles, R. K. (2013). "Abnormal Endocrine and Behavioural Sleep Markers in a Child with Williams Syndrome and Siblings," Journal of Sleep Disorders and Therapy, 2: 108.

Dunn, L. M., Dunn, L. M., Whetton, C. \& Burley, J. (1997). "British Picture Vocabulary Scale," Windsor, UK: NFER-Nelson.

Durmer, J. S. \& Dinges, D. F. (2005). "Neurocognitive Consequences of Sleep Deprivation," Seminars in Neurology, 25, (1), 117-129.
Dorfberger, S., Adi-Japha, E. \& Karni, A. (2007). "Reduced Susceptibility to Interference in the Consolidation of Motor Memory before Adolescence," Plos One, 2(2): E240.

Gais, S., Plihal, W., Wagner, U. \& Born, J. (2000). "Early Sleep Triggers Memory for Early Visual Discrimination Skills," Nature Neuroscience, 3(12), 1335-1339.

Gervan, P., Gombos, F. \& Kovacs, I. (2012). "Perceptual Learning in Williams Syndrome: Looking Beyond Averages," Plos ONE 7(7).

Goldman, S. E., Malow, B. A., Newman, K. D., Roof, E. \& Dykens, E. M. (2009). "Sleep Patterns and Daytime Sleepiness in Adolescents and Young Adults with Williams Syndrome," Journal of Intellectual Disability Research, 53(2), 182-188.

Jan, J. E., Owens, J. A., Weiss, M. D., Johnson, K. P., Freeman, R. D. \& Ipsiroglu, O. S. (2008). "Sleep Hygiene for Children with Neurodevelopmental Disabilities," Pediatrics, 122(6), 1343-1350.

Karni, A., Meyer, G., Jezzard, P., Adams, M. M., Turner, R. \& Ungerleider, L. G. (1995). "Functional MRI Evidence for Adult Motor Cortex Plasticity During Motor Skill Learning," Nature, 377, 155-158.

Maquet, P., Laureys, S., Peigneux, P., Fuchs, S., Petiau, C., Phillips, C., Aerts, J., Fiore, G. D., Degueldre, C. \& Meulemans, T. (2000). "Experience-Dependent Changes in Cerebral Activation During Human REM Sleep," Nature Neuroscience, 3(8), 831-836.

Mervis, C. B. \& Bertrand, J. (1997). 'Developmental Relations between Cognition and Language: Evidence from Williams Syndrome,' In L. B. Adamson and M. A. Romski (Eds.), Research on Communication and Language Disorders: Contributions to Theories of Language Development, 75-106. New York: Brookes. 
Mervis, C. B., Robinson, B. F., Bertrand, J., Morris, C. A., Klein-Tasman, B. P. \& Armstrong, S. C. (2000). "The Williams Syndrome Cognitive Profile," Brain and Cognition, 44, 604-628.

Morris, C. A., Demsey, S. A., Leonard, C. O., Dilts, C. \& Blackburn, B. L. (1988). "Natural History of Williams Syndrome: Physical Characteristics," Journal of Paediatrics, 113, 318-326.

Owens, J. A., Spirito, A. \& McGuinn, M. (2000). "The Children's Sleep Habits Questionnaire (CSHQ): Psychometric Properties of a Survey Instrument for School-Aged Children," Sleep. 23(8), 1-9.

Plihal, W. \& Born, J. (1997). "Effects of Early and Late Nocturnal Sleep on Declarative and Procedural Memory," Journal of Cognitive Neuroscience, 9(4), 534-547.

Pober, B. R. (2010). "Williams-Beuren Syndrome," New England Journal of Medicine, 362, 239-252.

Raven, J. C., Court, J. H. \& Raven, J. C. (1990). 'Manual for Raven's Progressive Matrices and Vocabulary Scales-Section 2: Coloured Progressive Matrices,' Oxford: Oxford Psychologists Press.

Richdale, A. \& Wiggs, L. (2005). "Behavioral Approaches to the Treatment of Sleep Problems in Children with Developmental Disorders: What Is State of the Art?," International Journal of Behavioral and Consultation Therapy, 1(3), 165-190.

Sadeh, A., Gruber, R. \& Raviv, A. (2003). "The Effects of Sleep Restriction and Extension on School-Age Children: What a Difference an Hour Makes," Child Development, 74(2), 44455.
Searcy, Y. M., Lincoln, A. J., Rose, F. E., Klima, E. S., Bavar, N. \& Korenberg, J. R. (2004). "The Relationship between Age and IQ in Adults with Williams Syndrome," American Journal on Mental Retardation, 109(3), 231-236.

Sniecinska, A. M., Iles, R. K., Butler, Stephen Andrew Butler, S. A., Jones, H., Bayford \& Dimitriou, D. (In Press) 'Sleep Disturbances, Elevated Cortisol and Low Melatonin in Children with Williams Syndrome,'

Thomas, M. S. C., Annaz, D., Ansari, D., Scerif, G., Jarrold, C. \& Karmiloff-Smith, A. (2009). "The Use of Developmental Trajectories in Studying Genetic Developmental Disorders," Journal of Speech, Language, and Hearing Research, 52, 336-358.

Walker, M. P., Brakefield, T., Morgan, A., Hobson, J. A. \& Stickgold, R. (2002). "Practice with Sleep Makes Perfect: Sleep-Dependent Motor Skill Learning," Neuron, 35, 205-211.

Walker, M. P., Brakefield, T., Seidman, J., Morgan, A., Hobson, J. A. \& Stickgold, R. (2003). "Sleep and the Time Course of Motor Skill Learning," Learning and Memory, 10(4), 275-284.

Waumans, R. C., Terwee, C. B., Van Den Berg, G., Knol, D. L., Litsenburg, R. R. L. \& Gemke, R. J. B. J. (2010). "Sleep and Sleep Disturbance in Children: Reliability and Validity of the Dutch Version of the Child Sleep Habits Questionnaire (CSHQ)," Sleep, 33:841-845. 